\section{0th Anniversary of PulseNet: the National Molecular Subtyping Network for Foodborne Disease Surveillance - United States, 2016}

PulseNet is celebrating 20 years of public health achievements in transforming the way foodborne disease outbreaks are detected and investigated. PulseNet is a national surveillance network of federal, state, and local public health laboratories that work together to detect foodborne disease outbreaks by connecting DNA fingerprints of bacteria that cause illness (1). The network facilitates the early identification of common sources of foodborne outbreaks and helps regulatory agencies identify areas where implementation of new measures are likely to improve the safety of the food supply.

A recent economic evaluation of PulseNet activities suggests that the network prevents at least 270,000 illnesses from infection with Salmonella, E. coli, and Listeria and saves an estimated $\$ 500$ million each year (2). In 2013, PulseNet began using whole genome sequencing (WGS) to detect outbreaks caused by Listeria, the most deadly foodborne pathogen (3). PulseNet is quickly expanding the use of WGS in state laboratories and has begun using WGS in investigations of other foodborne pathogens such as Campylobacter, E. coli, and Salmonella. With incorporation of WGS and other advanced molecular detection methods, PulseNet will continue to improve foodborne disease detection and identify outbreaks faster and with more accuracy. Additional information regarding CDC's Advanced Molecular Detection initiative is available at http://www. cdc.gov/amd/. Additional materials on the 20th anniversary of PulseNet, including success stories from state public health laboratories and fact sheets are available at the CDC PulseNet website.*

*http://www.cdc.gov/pulsenet/anniversary/index.html.

\section{References}

1. Swaminathan B, Barrett TJ, Hunter SB, Tauxe RV; CDC PulseNet Task Force. PulseNet: the molecular subtyping network for foodborne bacterial disease surveillance, United States. Emerg Infect Dis 2001;7:382-9. http:// dx.doi.org/10.3201/eid0703.017303

2. Scharff RL, Besser J, Sharp DJ, Jones TF, Peter GS, Hedberg CW. An economic evaluation of PulseNet: a network for foodborne disease surveillance. Am J Prev Med 2016;50(Suppl 1):S66-73. http://dx.doi. org/10.1016/j.amepre.2015.09.018

3. Jackson BR, Tarr C, Strain E, et al. Implementation of nationwide realtime whole-genome sequencing to enhance Listeriosis outbreak detection and investigation. Clin Infect Dis 2016. Epub April 18, 2016. 